Trabajos originales 


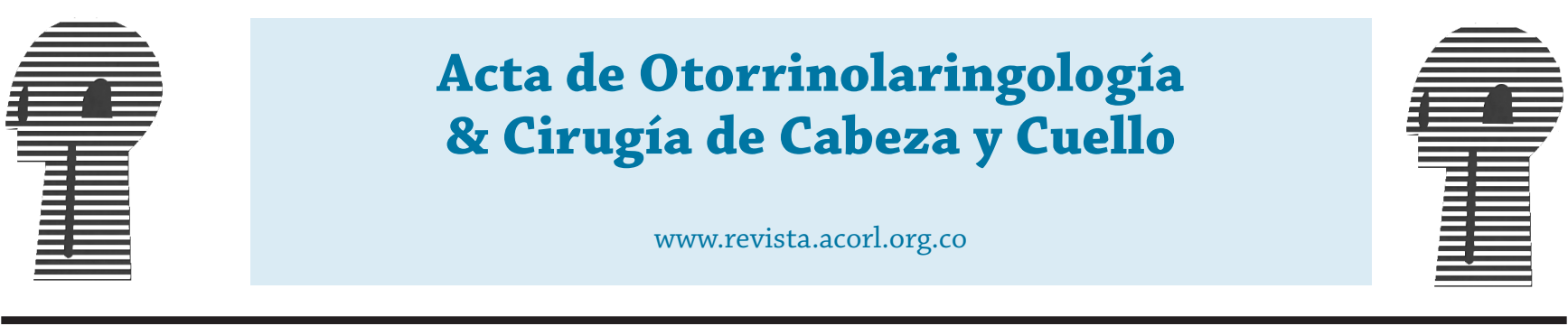

Trabajos originales

\title{
Características demográficas de la población con las principales patologías otorrinolaringológicas en Colombia
}

\section{Demographic characteristics of the population with the main ENT pathologies in Colombia}

\author{
Santiago Gutiérrez Maldonado*Elisa Gutiérrez-Gómez ${ }^{* *}$, Danielle Bastidas Rosas***. \\ * Otorrinolaringólogo, Hospital Universitario San Ignacio; Bogotá, Colombia. \\ ** Otorrinolaringóloga, Clínica de Marly; Bogotá, Colombia. \\ *** Médica general, Pontifica Universidad Javeriana; Bogotá, Colombia.
}

Forma de citar: Forma de citar: Gutiérrez-Maldonado S, Gutiérrez-Gómez E, Bastidas-Rosas D. Características demográficas de la población con las principales patologías otorrinolaringológicas en Colombia. Acta otorrinolaringol. cir. cabeza cuello. 2021;49(4): 272-278 DOI.10.37076/acorl.v49i4.608

\section{INFORMACIÓN DEL ARTÍCULO}

Historia del artículo:

Recibido: 13 de abril de 2021

Evaluado: 23 de noviembre 2021

Aceptado: 17 de diciembre 2021

Palabras clave (DeCS):

Otolaringología, epidemiología,

sistema respiratorio

\begin{abstract}
RESUMEN
Introducción: las patologías otorrinolaringológicas son de alta prevalencia en el mundo; en Colombia se han realizado pocos estudios sobre la frecuencia de estas patologías. A través del sistema integrado de protección social (SISPRO) y los códigos de Registros Individuales de Prestación de Servicios de salud (RIPS) es posible tener información oportuna, suficiente y estandarizada para la toma de decisiones en el sector de la salud. Objetivo: el objetivo del presente estudio fue realizar un análisis descriptivo de las principales características demográficas de la población con las principales patologías otorrinolaringológicas en Colombia. Resultados: se encontró que la rinitis alérgica y las patologías vertiginosas corresponden a las principales causas de consulta en este país, y las patologías infecciosas ocupan un papel importante especialmente en la población menor de 5 años. Adicionalmente, la gran mayoría de las patologías prevalentes en Colombia pueden ser manejadas en instituciones de primer nivel o atención primaria. Conclusión: es importante conocer la prevalencia de patologías en otorrinolaringología en Colombia para implementar campañas que divulguen su diagnóstico y tratamiento entre los médicos generales, médicos familiares y pediatras, con el fin de tener una adecuada cobertura a nivel nacional.
\end{abstract}




\section{ABSTRACT}

Key words (MeSH):

Otolaryngology; Epidemiology;

Respiratory System.
Introduction: ENT pathologies are highly prevalent in the world and few studies have been carried out in Colombia regarding this topic. Through the integrated social protection system (SISPRO by its acronym in Spanish) and the codes of Individual Registries for the Provision of Health Services (RIPS by its acronym in Spanish) it is possible to have standardized information for decision-making in the health sector. Objective: The purpose of this study was to carry out a descriptive analysis of the main demographic characteristics of the population with the main ENT pathologies in Colombia. Results: It was found that allergic rhinitis and vertiginous pathologies correspond to the main causes of consultation in this country and infectious pathologies occupy an important role in the population under 5 years of age. Additionally, the vast majority of the prevalent pathologies in Colombia can be managed in first-level institutions or primary care. Conclusions: It is important to know the prevalence of the main ENT pathologies in Colombia so we can implement campaigns that disseminate their diagnosis and treatment among general practitioners, family doctors and pediatricians in order to have adequate national coverage.

\section{Introducción}

Las patologías otorrinolaringológicas son de alta prevalencia en el mundo; existen estudios que han identificado que hasta el 52,2\% de los niños que consultan a urgencias tienen algún tipo de diagnóstico relacionado con la otorrinolaringología (1), mientras que otros estudios muestran que el $49 \%$ de las consultas de medicina general se asocian con la otorrinolaringología (2).

Colombia cuenta con un sistema integrado de protección social o SISPRO, herramienta creada y actualizada por el Ministerio de Salud y Protección Social (MSPS) con el fin de mantener bases de datos y sistemas de información en el sector salud que permitan tener información oportuna, suficiente y estandarizada para la toma de decisiones en el sector de la salud (3). Asociados con esta base de datos se encuentran los códigos RIPS (Registros Individuales de Prestación de Servicios de salud), los cuales, a su vez, guardan relación con los códigos diagnósticos del CIE-10 (Clasificación Internacional de Enfermedades) y son reportados directamente por los prestadores de atención a los usuarios. Estos códigos RIPS permiten obtener información demográfica, geográfica y de atenciones en salud (4). Teniendo en cuenta lo anterior, se hace posible la recuperación de datos nacionales, lo cual facilita el análisis secundario de esta información.

El objetivo de este estudio fue realizar un análisis descriptivo de las principales características demográficas de la población con las principales patologías otorrinolaringológicas en Colombia.

\section{Materiales y métodos}

Se tomaron los datos del SISPRO teniendo en cuenta los códigos RIPS asociados con los principales diagnósticos vistos en otorrinolaringología.

El ingreso a SISPRO se realizó mediante una tabla dinámica de Microsoft Excel, a la cual se pudo acceder luego de haber realizado los trámites exigidos por el MSPS. La información obtenida se procesa en línea en tablas dinámicas que permiten recolectar información sobre diferentes variables como diagnóstico principal, edad de atención, fecha de atención, entre otros. Para el análisis de las patologías se utilizaron como criterios de inclusión los siguientes códigos CIE-10 registrados entre 2015 a 2019 (Tabla 1). Gran parte de la información recogida en los RIPS se excluyó y no fue considerada para el análisis, ya que se sale del objetivo del presente artículo.

Se realizó un análisis de las bases de datos de SISPRO, incluyendo las consultas de urgencias y consulta externa, con el fin de determinar los principales motivos de consulta en otorrinolaringología en Colombia durante cinco años, entre el primero de enero de 2015 al 31 de diciembre de 2019.

\section{Resultados}

Se encontró un total de eventos de 11719391 en el período observado; dentro de este, los principales motivos de consulta encontrados incluyen: rinitis con 3958363 atenciones $(33,7 \%)$, patología vertiginosa que corresponde a 3596565 atenciones $(30,6 \%)$ y patologías infecciosas, dentro de las que se encuentran amigdalitis, otitis media y sinusitis, para un total de 3569716 (30,4\%) (Tabla 2).

Las cinco patologías infecciosas más frecuentes como motivo de consulta de Otorrinolaringología incluyen, en orden de presentación, faringitis, amigdalitis, otitis media, otitis externa y laringitis. El grupo de edad que mayor presenta patologías infecciosas es de 1 a 5 años, seguido por los pacientes entre 27 y 44 años. El principal motivo de consulta corresponde a faringitis, principalmente en el grupo de 1 a 5 años, y con un segundo pico entre 27 y 44 años, seguido por amigdalitis con una prevalencia baja en el grupo de 0 a 1 año. El tercer motivo de consulta en el grupo de patologías infecciosas corresponde a otitis media con un mayor número de casos presentándose en el grupo entre 1 a 5 años (Figura 1). 
Tabla 1. Diagnósticos y sus respectivos códigos CIE-10 Diagnóstico

\begin{tabular}{|c|c|}
\hline Diagnóstico & Código CIE-10 \\
\hline Microtia & Q170-175 y Q181 \\
\hline Patología de mastoides & H71X, H700-702, H708-H709 \\
\hline $\begin{array}{l}\text { Otoesclerosis y } \\
\text { timpanoesclerosis }\end{array}$ & $\mathrm{H} 800-809, \mathrm{H} 740$ \\
\hline Hipoacusia & H900-908, H910, H912, H918-19 \\
\hline $\begin{array}{l}\text { Patologías de membrana } \\
\text { timpánica }\end{array}$ & H720-722, H728-32, H738-39 \\
\hline Otitis externa & $\mathrm{H} 600-603, \mathrm{H} 605, \mathrm{H} 608-10, \mathrm{H} 620-24$ \\
\hline Otitis media & $\begin{array}{l}\mathrm{H} 650-54, \mathrm{H} 659-64, \mathrm{H} 667, \mathrm{H} 670-71 \text {, } \\
\mathrm{H} 678\end{array}$ \\
\hline $\begin{array}{l}\text { Patología de trompa de } \\
\text { Eustaquio }\end{array}$ & H680-81, H690, H698, H699 \\
\hline Patología de laringe & $\begin{array}{l}\text { A545, J00, J020, J028-29, J060, } \\
\text { J311-12 }\end{array}$ \\
\hline Laringitis & $\begin{array}{l}\text { J040, J050, J370, J042, J052, } \\
\text { J060 }\end{array}$ \\
\hline Amigdalitis & $\begin{array}{l}\text { B002, J030, J038-39, J350, J020, } \\
\text { J028 }\end{array}$ \\
\hline $\begin{array}{l}\text { Fractura de huesos propios } \\
\text { nasales }\end{array}$ & S022 \\
\hline Epistaxis & R040 \\
\hline Desviación septal & J342 \\
\hline Sinusitis aguda & J010-14, J018-19 \\
\hline Sinusitis crónica & J320-24, J328-29 \\
\hline Patología tumoral & $\begin{array}{l}\text { C300, C318-19, D140, J330-31, } \\
\text { J338-39, J341, C320-23, C328, } \\
\text { C141. }\end{array}$ \\
\hline Rinitis & $\mathrm{J} 300-304, \mathrm{~J} 310$ \\
\hline Vértigo & $\begin{array}{l}\text { H810-14, H 818-19, H82X, H830, } \\
\text { H832 }\end{array}$ \\
\hline
\end{tabular}

Tabla 2. Patologías con mayor número total de personas atendidas durante 2015-2019

\begin{tabular}{|l|c|c|}
\hline \multicolumn{1}{|c|}{ Patología } & Número de personas & Porcentaje (\%) \\
\hline Rinitis & $\mathrm{N}=3958363$ & 33,78 \\
\hline Vértigo & $\mathrm{N}=3596565$ & 30,69 \\
\hline Faringitis & $\mathrm{N}=1.883 .605$ & 16,07 \\
\hline Amigdalitis & $\mathrm{N}=852.184$ & 7,27 \\
\hline Otitis media & $\mathrm{N}=323.941$ & 2,76 \\
\hline Otoesclerosis & $\mathrm{N}=202.549$ & 1,73 \\
\hline Hipoacusia & $\mathrm{N}=201.239$ & 1,72 \\
\hline Sinusitis aguda & $\mathrm{N}=173.429$ & 1,48 \\
\hline Laringitis & $\mathrm{N}=155.771$ & 1,33 \\
\hline Otitis externa & $\mathrm{N}=117.127$ & 1,00 \\
\hline Epistaxis & $\mathrm{N}=68.117$ & 0,58 \\
\hline Sinusitis crónica & $\mathrm{N}=63.659$ & 0,54 \\
\hline Desviación septal & $\mathrm{N}=45.904$ & 0,39 \\
\hline Parálisis facial & $\mathrm{N}=23208$ & 0,20 \\
\hline Membrana & $\mathrm{N}=12.305$ & 0,10 \\
\hline timpánica & & \\
\hline
\end{tabular}

En total se encontraron 3958363 consultas a urgencias o consulta externa por rinitis durante los años 2015-2019. Este es el principal motivo de consulta en las patologías otorrinolaringológicas en este período (Figura 2). Dentro de la rinitis, el principal motivo de consulta fue por rinitis alérgica no especificada, seguida de otras rinitis alérgicas y rinitis crónica. $\mathrm{Al}$ analizar por grupos de edad, se encuentra un pico de presentación entre los primeros cinco años y otro entre los 27 a 44 años (Figura 3). En todos los grupos de edad, el principal motivo de consulta fue por rinitis alérgica no especificada seguida de otras rinitis alérgicas. Asimismo, se encontró una muy baja prevalencia de rinitis vasomotora, rinitis alérgica debida al polen u otra rinitis alérgica estacional. Al hacer un análisis de las principales patologías de oído, nariz y laringe encontramos que, en cuanto a patologías de oído, el principal motivo de consulta corresponde a otitis media con un total de 1619704 atenciones, para un promedio de 323940 atenciones por año, seguido de la otosclerosis para un total de 1012744 atenciones en los cinco años analizados e hipoacusia como tercer motivo de consulta para un total de 1006196 consultas. La parálisis facial se encontró como quinto motivo de consulta en patologías de oído precedida por la otitis externa (Figura 4).

En cuanto a las patologías nasales, el principal motivo de consulta fue por rinitis alérgica; no obstante, el segundo motivo de consulta fue por sinusitis aguda, la cual tuvo una mayor prevalencia entre 27 a 44 años, seguida por el grupo de edad de 19 a 26 años. Otros motivos de consulta menos frecuentes fueron la epistaxis con un total de 340587 consultas en los cinco años analizados y un pico de presentación en el grupo de edad de mayores de 60 años y la desviación septal con un total de 229519 consultas con una presentación mayor entre 27 a 44 años.

Finalmente, en cuanto a patologías de faringolaringe se encontró la faringitis como el principal motivo de consulta en todas las edades con un total de 9418016 en los cinco años analizados, con un promedio de 1883603 casos al año y con mayor frecuencia para el grupo de edad de 1 a 5 años, seguido de 27 a 44 años; luego por amigdalitis para un total de 4260920 casos, con un promedio de 852184 consultas al año, con mayor presentación entre 27 a 44 años, seguido por el grupo de edad de 1 a 5 años. Al revisar patologías infecciosas, se encuentra que la mayoría de estas se presentan en el grupo de uno a cinco años de edad, seguida por 27 a 44 años, siendo en ambos grupos la faringitis la más prevalente, seguida de amigdalitis y en tercer puesto la otitis media (Figura 5).

En cuanto a la patología tumoral, se encontró que el principal grupo de presentación se encuentra entre 1 a 5 años, seguido de 27 a 44 años y 45 a 59 años; sin embargo, este grupo se cataloga como tumores y no especifica el tipo de tumor que presenta cada edad. 


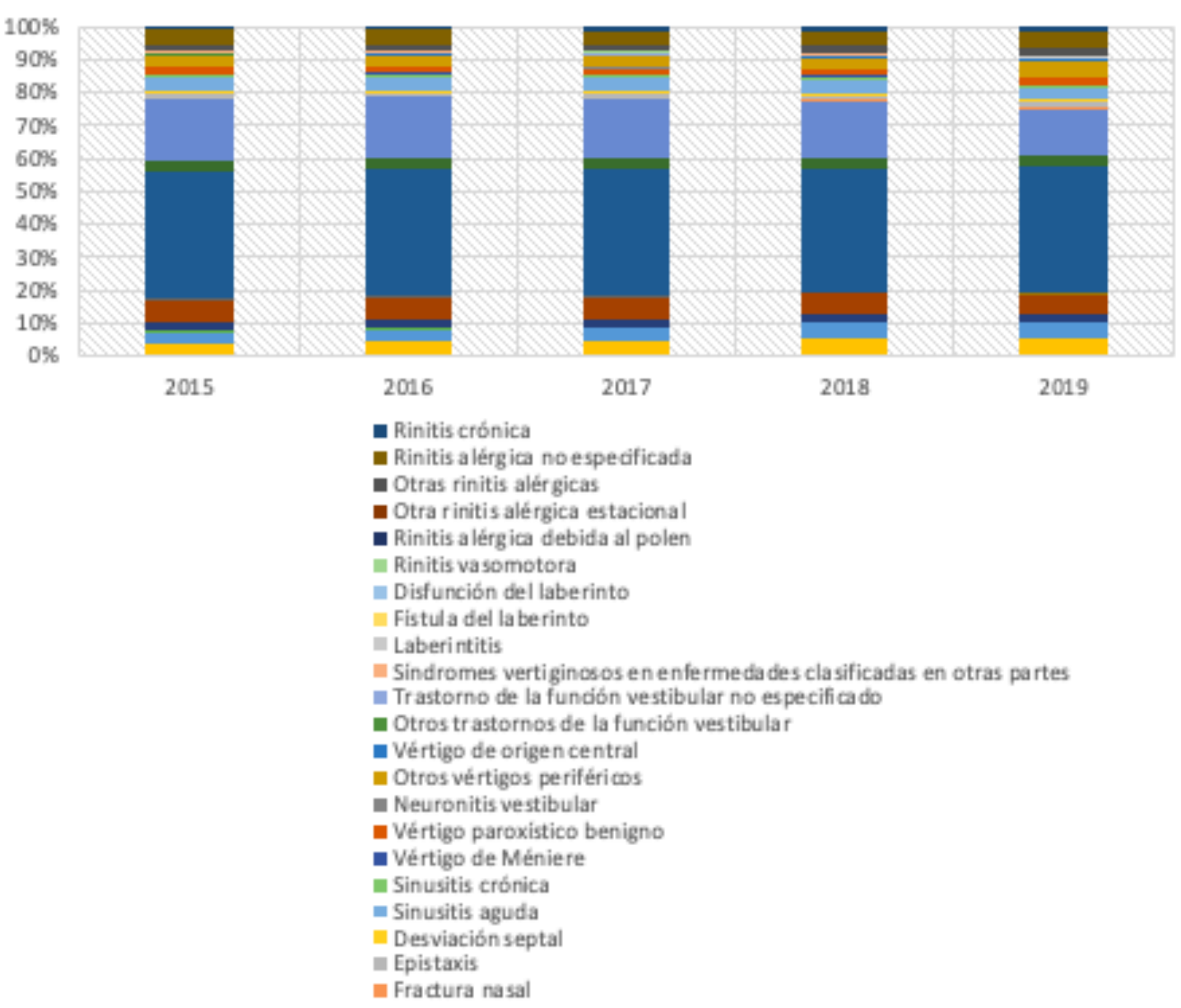

Figura 1. Frecuencia acumulada de las patologías más frecuentes en otorrinolaringología (ORL). Fuente: Elaboración propia

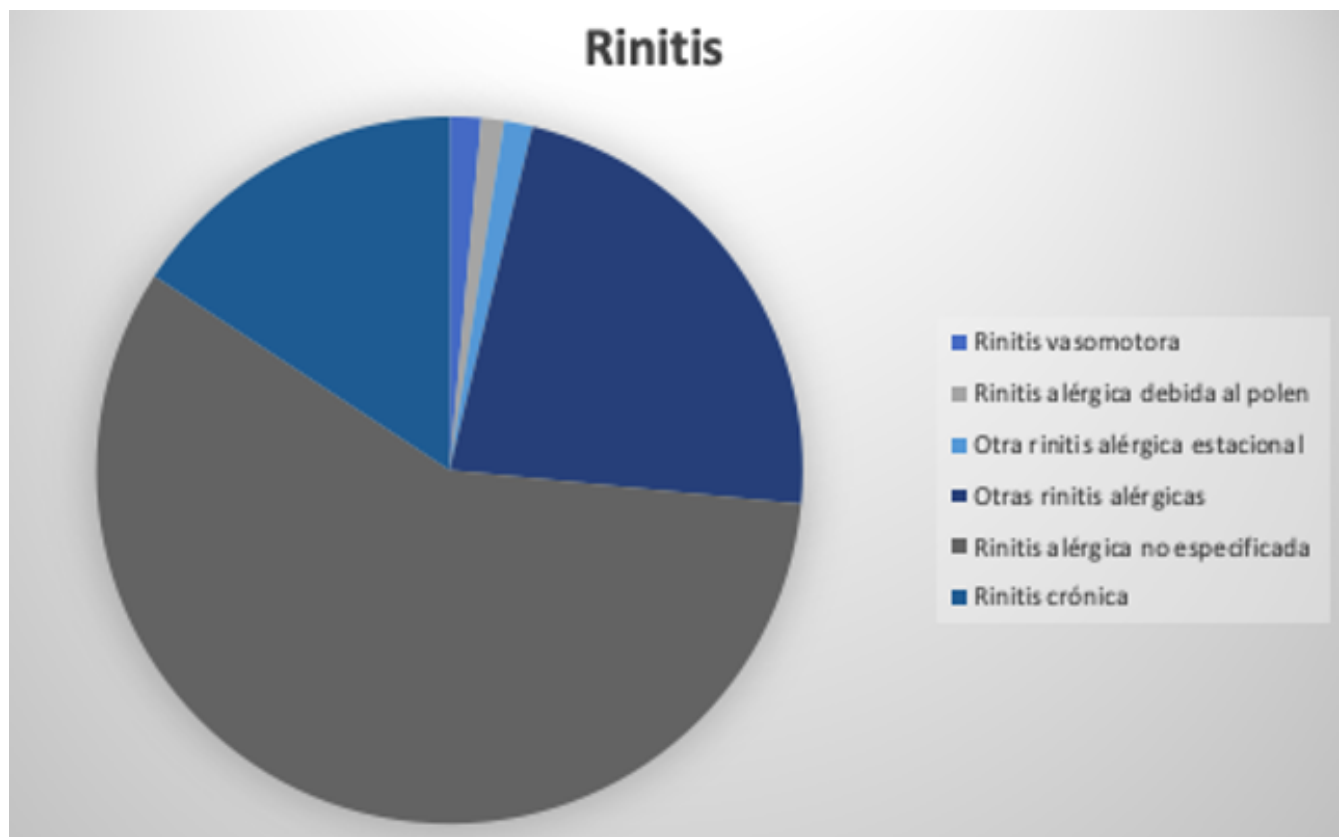

Figura 2. Porcentaje de casos de las diferentes patologías asociadas con la rinitis alérgica. Fuente: Elaboración propia 
Distribución de rinitis por grupo de edad

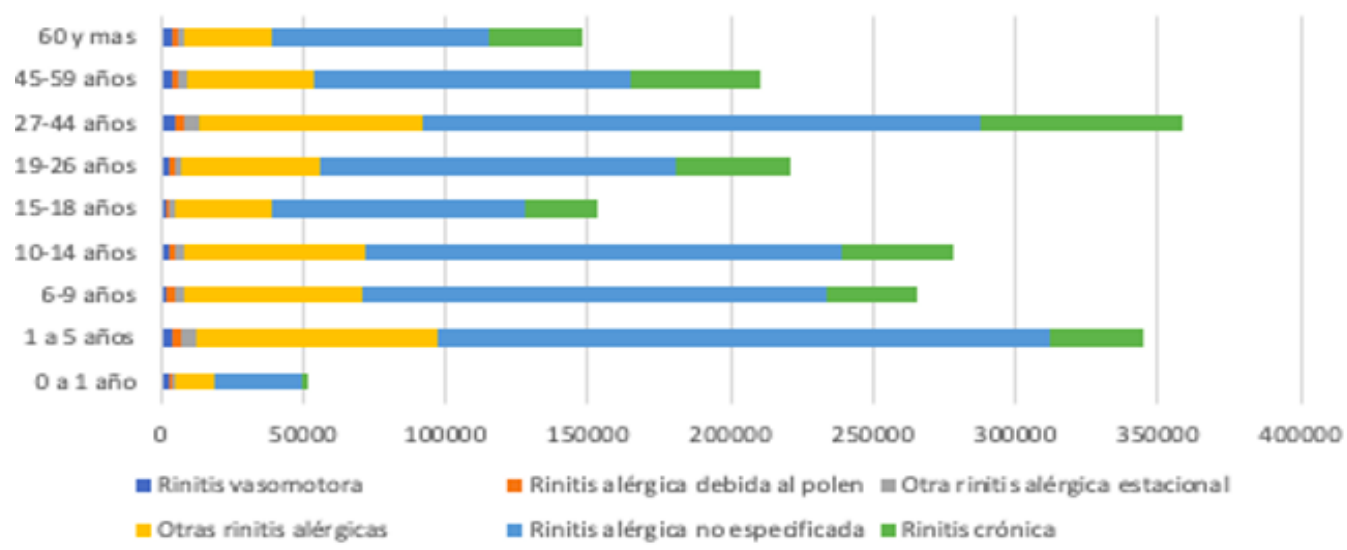

Figura 3. Número de casos de rinitis alérgica por grupo etario. Fuente: Elaboración propia

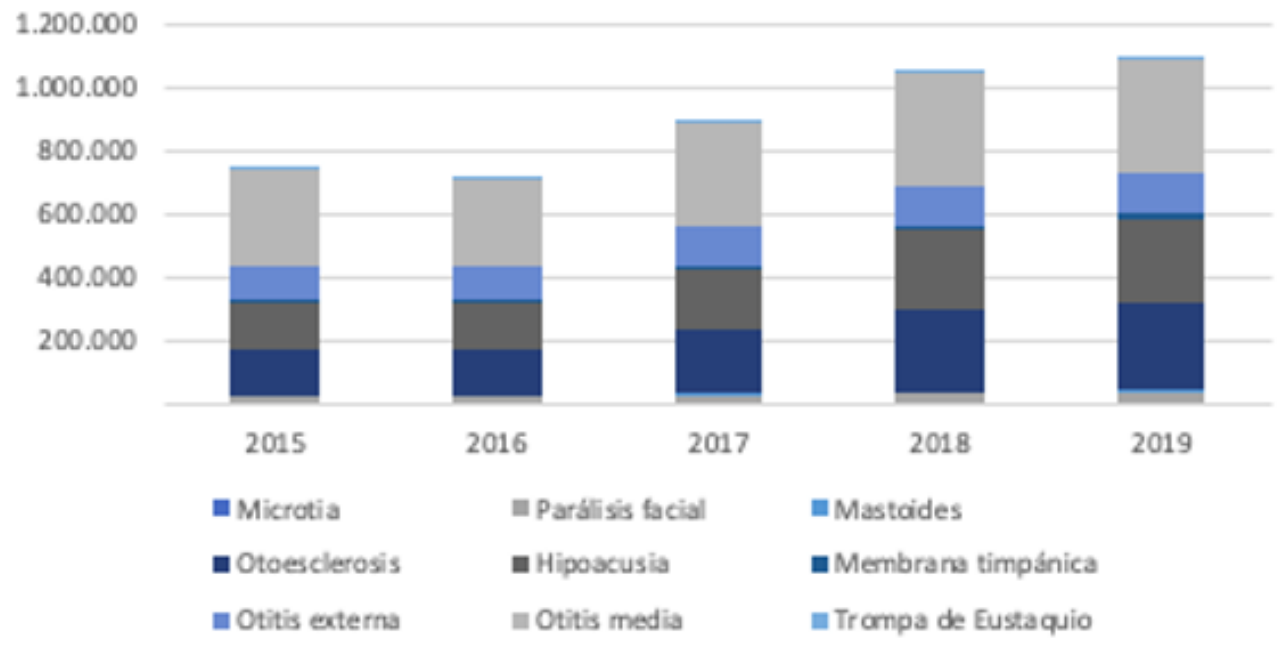

Figura 4. Principales patologías de oído. Fuente: Elaboración propia

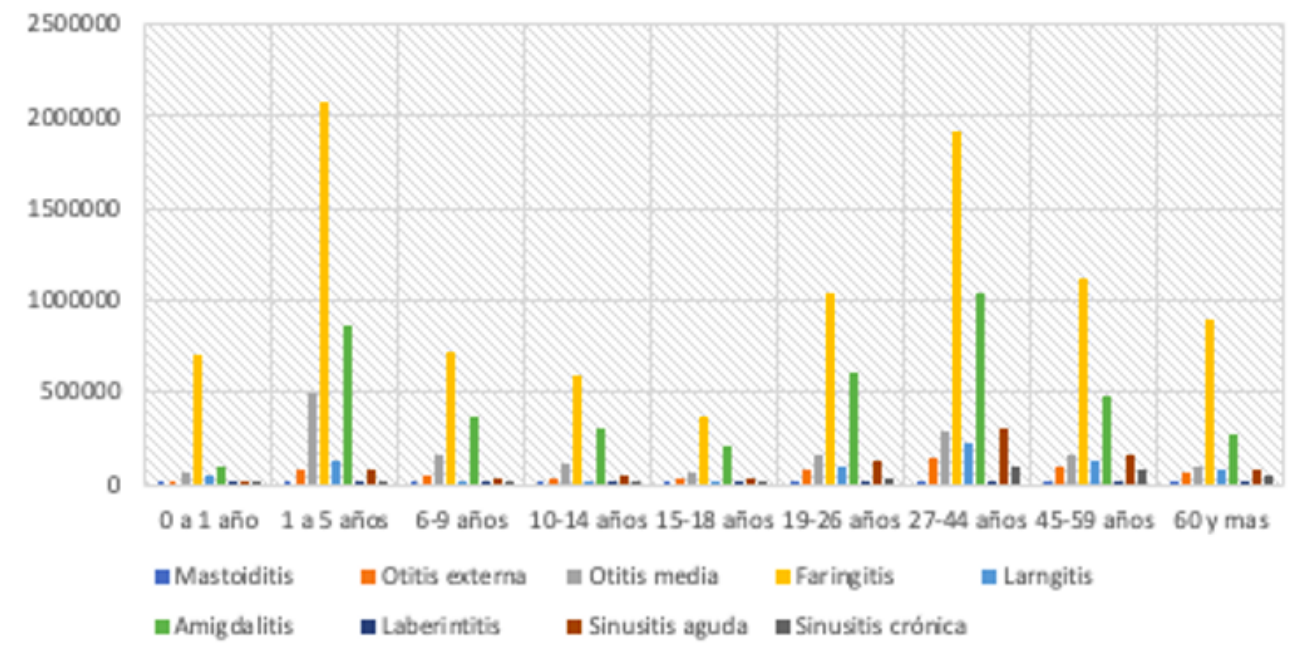

Figura 5. Principales patologías infecciosas según grupo de edad. Fuente: Elaboración propia 


\section{Discusión}

En Colombia se han realizado pocos estudios sobre la frecuencia de patologías otorrinolaringológicas. Sin embargo, gracias al SISPRO se cuenta con bases de datos y sistemas de información en el sector de la salud que permitan tener información oportuna, suficiente y estandarizada; esto posibilita la realización de un análisis histórico sobre los principales motivos de consulta, tanto en urgencias como en consulta externa, de las patologías otorrinolaringológicas en nuestro país.

$\mathrm{Al}$ realizar un análisis de los datos, se encuentra que los principales motivos de consulta en otorrinolaringología en nuestro país entre 2015 y 2019 incluyen rinitis, patología vertiginosa y patologías infecciosas. Los grupos de edad que presentan mayor asistencia a urgencias o consulta externa son los pacientes entre 1 y 5 años, con un segundo pico entre los 27 y 44 años, lo que coincide con algunos reportes de la literatura donde este grupo de edad representa la mayor cantidad de consultas (5-7). En ambos casos, la patología más frecuente de consulta es la rinitis alérgica, con una alta frecuencia también de rinitis crónica, aunque cabe anotar que estos diagnósticos pueden ser realizados tanto por medicina general, pediatría, medicina familiar u otorrinolaringología (4). Esto hace que se generen diagnósticos generales sin especificar claramente el tipo de rinitis que presenta el paciente. Este resultado contrasta con algunos reportes en nuestro país y en la literatura mundial, donde se encuentran las patologías de oído como principales motivos de consulta, seguidos de patologías de nariz, faringe y laringe (8-11).

Con respecto a las patologías infecciosas, se ha encontrado que la más frecuente son la faringitis, la amigdalitis y la otitis media, y que dichas enfermedades son más frecuentes en la población menor de 5 años (Figura 5). Dicha información se ve respaldada en la literatura en la que estas se encuentran como causas frecuentes de enfermedades infecciosas en otorrinolaringología en niños $(4,12,13)$. Es posible que, como se había mencionado anteriormente, las patologías de otorrinolaringología puedan ser vistas por medicina general, medicina familiar o pediatría, e incluye todos los diagnósticos del CIE-10 asignados por estos profesionales, por lo que se puede atribuir un código general que no corresponde al diagnóstico específico del paciente.

Llama la atención la alta prevalencia de otosclerosis que supera incluso diagnósticos como hipoacusia o parálisis facial, los cuales son altamente frecuentes en la literatura mundial e incluso en la consulta de otorrinolaringología. Es probable que se haga un diagnóstico errado de estas patologías o se use un código incorrecto para diagnosticar patologías de oído sin que sea este el verdadero diagnóstico, lo que no nos permite calcular de forma precisa estas frecuencias. Es parecido lo que ocurre con diagnósticos como epistaxis o desviación septal cuyas frecuencias en este estudio son mucho más bajas de lo que se reporta en la literatura mundial $(14,15)$. Según el último estudio publicado en 2020 por Mors y colaboradores, la epistaxis fue el principal motivo de consulta (16-18) y es considerado, en algunos reportes, como uno de los principales motivos de consulta a urgencias, seguido de la otitis externa, otitis media aguda y vértigo $(5,8,15)$.

Es importante también anotar que existen códigos del CIE-10 que pueden usarse para patologías similares, por ejemplo, H651- (otra otitis media aguda no supurativa) y H659- (otitis media no supurativa) sin otra especificación y que generan una confusión en el momento de realizar el análisis epidemiológico de estas patologías, ya que al sumarlos cambiaría la prevalencia de algunas enfermedades.

Cabe anotar que el sistema integrado de protección social (SISPRO) ha permitido generar una base de datos fundamental para el estudio de patologías prevalentes en Colombia en diferentes especialidades, aunque, como se ha anotado previamente, debe tenerse consideraciones en cuanto a sus resultados por las limitaciones antes mencionadas. Este trabajo nos permite orientar las principales patologías otorrinolaringológicas por las que se consulta actualmente en Colombia, encontrando la alta prevalencia de consulta por patologías infecciosas y patologías de nariz.. . Adicionalmente, la mayoría de las patologías, según lo reportado, pueden ser vistas por médicos generales, medicina general o pediatría, lo que permite una mayor cobertura a nivel nacional, siendo muy importante la remisión a otorrinolaringología para patologías de mayor complejidad.

\section{Conclusiones}

Las patologías otorrinolaringológicas son de alta prevalencia en el mundo y en Colombia. En la actualidad encontramos que la rinitis alérgica y patologías vertiginosas corresponden a las principales causas de consulta en este país. Las patologías infecciosas también ocupan un lugar importante, sobre todo en la población infantil menor de 5 años. La mayoría de las patologías previamente mencionadas pueden ser manejadas en instituciones de primer nivel o atención primaria, por lo que es necesario implementar campañas que divulguen su diagnóstico y tratamiento entre los médicos generales, médicos familiares y pediatría con el fin de tener una adecuada cobertura a nivel nacional.

El sistema integrado de protección social (SISPRO) es una gran herramienta que permite el análisis de grandes bases de datos a nivel nacional y cuyo uso puede ayudar al estudio epidemiológico de las patologías en Colombia.

\section{Conflictos de interés}

Los autores de este artículo declaran no tener conflictos de interés.

\section{Agradecimientos}

Agradecemos a la Dra. Isabel del Socorro Moreno por su apoyo en la corrección de este artículo. 


\section{Aspectos éticos}

Se considera un estudio de bajo riesgo. Se realizó un estudio de los datos de forma anonimizada y no es posible identificar a ninguna persona a partir de los datos analizados.

\section{REFERENCIAS}

1. Signorelli LG, Mendes EdeA. Prevalence of otorhinolaryngologic diagnoses in the pediatric emergency room. Int Arch Otorhinolaryngol. 2013;17(1):10-3. doi: 10.7162/S180997772013000100002

2. Díaz-Ledesma L, Dávila-Acosta JH, Rodríguez-Salazar VM, Añaños-Flores DM. Frecuencia de diagnósticos de la especialidad de otorrinolaringología en el consultorio de medicina general en un centro de salud. Rev Med Hered. 2003;14(4):163-6.

3. Ministerios de Salud de Colombia [Internet]. Sispro.gov. co; 2020. SISPRO - Sistema Integrado de Información de la Protección Social. [Consultado el 23 de diciembre de 2020]. Disponible en: https://www.sispro.gov.co/Pages/Home.aspx

4. Ministerios de Salud de Colombia [Internet]. Minsalud.gov. co; 2020. Sistema De Información De Prestaciones De Salud RIPS. [Consultado el 23 de diciembre de 2020]. Disponible en: https://www.minsalud.gov.co/proteccionsocial/Paginas/rips. aspx

5. Yojana S, Mehta K, Girish M. Epidemiological profile of otorhinolaryngological emergencies at a medical college, in rural area of gujarat. Indian J Otolaryngol Head Neck Surg. 2012;64(3):218-24. doi: 10.1007/s12070-011-0293-8

6. Hannaford PC, Simpson JA, Bisset AF, Davis A, McKerrow W, Mills R. The prevalence of ear, nose and throat problems in the community: results from a national cross-sectional postal survey in Scotland. Fam Pract. 2005;22(3):227-33. doi: 10.1093/fampra/cmi004

7. Ruz GS, Breinbauer KH,Arancibia SM. Análisis epidemiológico de la patología otorrinolaringológica ambulatoria en el Hospital San Juan de Dios. Rev. Otorrinolaringol. Cir. Cabeza Cuello. 2009;69(3):227-32. doi: 10.4067/S0718-48162009000300004

8. Raj A, Wadhwa V, Jain A. Epidemiological Profile of ENT Emergencies: Our Experience. Indian J Otolaryngol Head Neck Surg. 2019;71(Suppl 1):301-4. doi: 10.1007/s12070-018-12849

9. Pino V, Trinidad G, González A, Pardo G, Pantoja CG, Marcos $M$, et al. Consideraciones sobre las urgencias ORL. Análisis de 30.000 pacientes atendidos en 10 años. Acta Otorrinolaringológica Española. 2005;56(5):198-201. doi:10.1016/S0001-6519(05)78600-3

10. Álvarez-Hernández LF, Martínez-Sánchez LM, JaramilloJaramillo LI. Enfermedades otorrinolaringológicas: una mirada genética. Archivos de Medicina (Col). 2017;17(1):142-9.

11. Jaramillo-Jaramillo L, Martínez-Sánchez L, Álvarez-Hernández L, Rodríguez-Gazquez M. Perfil clínico y epidemiológico de pacientes de consulta externa ORL en una institución de salud de mediana complejidad de Antioquia, Colombia, 2010-2014. Revista médica de Panamá. 2016;36(3):10-14.

12. Choi KJ, Kahmke RR, Crowson MG, Puscas L, Scher RL, Cohen SM. Trends in Otolaryngology Consultation Patterns at an Academic Quaternary Care Center. JAMA Otolaryngol Head Neck Surg. 2017;143(5):472-7. doi: 10.1001/ jamaoto.2016.4056

13. Signorelli LG, Mendes EA. Prevalence of otorhinolaryngologic diagnoses in the pediatric emergency room. Int. Arch.
Otorhinolaryngol. 2013;17(1):10-13 [Nota: esta referencia se repite con la 1; por favor indicar si se debe reemplazar por otra o se debe eliminar y reenumerar la lista de referencias] - Esta referencia debe eliminarse.

14. Rojas-Roncancio E, Peña-Silva R. Epidemiología digital como herramienta para la evaluación de enfermedades del oído y la vía aérea en Colombia, durante los años 2012-2014: Un estudio ecológico. Acta otorrinolaringol cir cabeza cuello. 2018;46(3):202-7. doi: 10.37076/acorl.v46i3.423

15. Vaduva C, Gómez JIT, Zaid DM, Rivera-Rodríguez T. Patología infecciosa aguda de foco otorrinolaringológico [Acute infectious disease of otolaryngology focus]. Medicine (Madr). 2019;12(91):5339-51. Spanish. doi: 10.1016/j. med.2019.11.021

16. Zeeshan M, Zeb J, Saleem M, Zaman A, Khan A, Tahir M. ENT diseases presenting to a tertiary care hospital. Endocrinol Metab Int J. 2018;6(6):416-41. doi: 10.15406/emij.2018.06.00225

17. Mors M, Bohr C, Fozo M, Shermetaro C. Consultation Intervention Rates for the Otolaryngology Service: A Large Metropolitan Hospital Experience. Spartan Med Res J. 2020;4(2):11596. doi: 10.51894/001c.11596

18. Lammens F, Lemkens N, Laureyns G, Lemmens W, Van Camp L, Lemkens P. Epidemiology of ENT emergencies. B-ENT. 2014;10(2):87-92. 